\title{
REVIEW ARTICLE \\ Role of environmental poliovirus surveillance in global polio eradication and beyond
}

\author{
T. HOVI ${ }^{1 *}$, L. M. SHULMAN ${ }^{2}$, H. VAN DER AVOORT ${ }^{3}, J_{\text {J DESHPANDE }}^{4}$, \\ M. ROIVAINEN ${ }^{1}$ AND E. M. DE GOURVILLE \\ ${ }^{1}$ National Institute for Health and Welfare (THL), Helsinki, Finland \\ ${ }^{2}$ Central Virology Laboratory (CVL), Ministry of Health, Sheba Medical Center, Tel-Hashomer, Israel \\ ${ }^{3}$ National Institute of Public Health and the Environment (RIVM), Bilthoven, The Netherlands \\ ${ }^{4}$ Enterovirus Research Centre (ERC), Mumbai, India \\ ${ }^{5}$ Global Poliomyelitis Eradication Initiative, WHO, Geneva, Switzerland
}

(Accepted 21 December 2010; first published online 18 August 2011)

\section{SUMMARY}

Environmental poliovirus surveillance (ENV) means monitoring of poliovirus (PV) transmission in human populations by examining environmental specimens supposedly contaminated by human faeces. The rationale is based on the fact that PV-infected individuals, whether presenting with disease symptoms or not, shed large amounts of PV in the faeces for several weeks. As the morbidity:infection ratio of $\mathrm{PV}$ infection is very low, this fact contributes to the sensitivity of ENV which under optimal conditions can be better than that of the standard acute flaccid paralysis (AFP) surveillance. The World Health Organization has included ENV in the new Strategic Plan of the Global Polio Eradication Initiative for years 2010-2012 to be increasingly used in PV surveillance, supplementing AFP surveillance. In this paper we review the feasibility of using ENV to monitor wild PV and vaccine-derived PV circulation in human populations, based on global experiences in defined epidemiological situations.

Key words: Infectious disease epidemiology, polio, polio vaccine virus, public health, surveillance.

\section{BACKGROUND}

\section{Success and remaining challenges of the Global Polio Eradication Initiative (GPEI)}

The GPEI, launched at the World Health Assembly (WHA) in 1988, is the single largest, internationally coordinated public health project the world has ever known [1]. It is spearheaded by national governments, the World Health Organization (WHO), Rotary International, the US Centers for Disease Control and Prevention (CDC) and UNICEF. Administration

\footnotetext{
* Author for correspondence: Professor T. Hovi, National Institute for Health and Welfare (THL), Mannerheimintie 166, PO Box 30, Helsinki 00271, Finland.

(Email: tapani.hovi@thl.fi)
}

of poliovirus (PV) vaccine to every child had been recommended by WHO since the 1970s in the context of the Expanded Programme of Immunizations (EPI) but the coverage never reached sufficiently high levels to stop PV circulation globally. In contrast, exploiting the key principles of GPEI [1, 2], especially together with the designated supplementary immunization activities (SIA, Table 1), rapidly changed the situation. Although the initially planned deadline of eradication, the year 2000 , is well in the past, the success of the programme has been marked (Table 2). The number of new cases has been reduced by more than $99 \%$ and no cases due to wild-type poliovirus 2 (WPV2) have been identified since 1999 [5, 6]. Furthermore, genetic diversity of the remaining WPV of serotypes 1 
Table 1. Original principles and current objectives of the Global Polio Eradication Initiative

Original key principles [2] Current key objectives [3]

(1) High coverage routine immunization of newborns with the oral poliovirus vaccine (OPV)

(2) Active surveillance for and standardized investigation of patients with acute flaccid paralysis (AFP) including virological analysis of faecal samples to detect polioviruses

(3) Supplementary immunization activities (SIA) including National Immunization days and house-to-house targeted mop-up rounds to rapidly boost immunity to polioviruses in children aged $<5$ years
(1) Interrupting wild poliovirus transmission in Asia

(2) Interrupting wild poliovirus transmission in Africa

(3) Enhancing global surveillance and outbreak response

(4) Strengthening immunization systems

Table 2. Selected indicators of the success of the Global Polio Eradication Initiative

\begin{tabular}{llllll}
\hline \hline Parameter & 1988 & 2000 & 2006 & 2009 & Ref. \\
\hline No. of new cases per year & 300000 & 719 & 1997 & 1606 & $*$ \\
No. of endemic countries & $>125$ & 20 & 4 & 4 & $*$ \\
No. of endemic WHO Regions & 6 & 3 & 3 & 3 & $*$ \\
No. of circulating wild WPV serotypes & 3 & 2 & 2 & 2 & $*$ \\
No. of remaining WPV genotypesं & WPV1: 20 & WPV1: 9 & WPV1:2 & WPV1:2 \\
& WPV2: 5 & WPV2:0 & WPV2:0 & WPV2:0 \\
& WPV3: 17 & WPV3: & WPV3:2 & WPV3:2 \\
\hline \hline
\end{tabular}

WPV, Wild-type poliovirus.

Since 1988, >2 billion children around the world have been immunized against polio, thanks to the unprecedented cooperation of more than 200 countries and 20 million volunteers, backed by an international investment of more than US\$ 5 billion.

* (http://www.polioeradication.org/content/general/casecount.pdf).

$\dagger$ A genotype is a group of genetically closely related poliovirus strains (difference in capsid protein VP1 coding sequence $\leqslant 15 \%$ ) that are considered to have epidemiological linkage with each other [4].

† O. Kew, personal communication.

and 3 has strongly decreased, indicating limited circulation. Since the start of the millennium, fewer than 2000 cases caused by WPVs have been reported annually.

In spite of the success, obstacles still confront the desired target. Re-emergence of WPV poliomyelitis in many previously polio-free countries demonstrates that herd immunity in some polio-free developing countries may be extremely fragile [6]. Even temporary deficits in vaccination coverage can sometimes be sufficient to facilitate circulation of imported WPV [7]. At the dawn of the millennium, yet another obstacle for global eradication of poliomyelitis was recognized: vaccine-derived polioviruses (VDPVs), genetically drifted strains that regain neurovirulence, have caused several outbreaks of paralytic poliomyelitis in different parts of the world [8].

The standard approach recommended by WHO for polio surveillance is the detection and investigation of cases of acute flaccid paralysis (AFP), which includes standardized virological analysis of two faecal samples of the patient, and/or sometimes those from contacts [2]. Although AFP surveillance is in principle applicable to any human population at any time, there are situations in which there are good reasons to suspect that negative results of AFP surveillance are not reliable. Supplementary information is required in such situations and one approach for that is environmental surveillance (ENV), in which a search for PV is made in environmental specimens contaminated by human faeces [9]. 


\section{ENV OF PV CIRCULATION}

\section{Rationale of ENV is based on the natural course of PV infection}

It is well known that the "typical paralytic case of poliomyelitis' is actually an exception in the natural course of PV infection: more than $99 \%$ of PV infections proceed without paralysis. Irrespective of the presence or absence of clinical symptoms, PV replication is considered to continue in the para-intestinal submucosal lymphatic tissue from several weeks to a few months. The virus is excreted into the faeces and shed into the environment. This forms the rationale for environmental PV surveillance. PV is also known to be excreted in the nasopharyngeal mucosa during the first 2 weeks of infection. This is considered to have a role in virus transmission between close contacts but is not likely to contribute strongly to the load of virus in the environment. The amount of virus excreted into stools is known to be variable with maximal amounts reaching $10^{7}$ infectious doses/day per person [10]. PVs are relatively stable in aqueous environments at ambient temperatures and adsorption to various solid materials in the environment may further extend the time over which at least part of the infectivity can be recovered. On the other hand, industrial waste, and in tropical countries, increase of temperature and UV irradiation from sunlight inactivate the virus in a time-dependent manner, a fact that has to be taken in account in designing ENV. In short, ENV offers an anonymous, non-invasive approach for monitoring $\mathrm{PV}$ circulation in populations at risk.

\section{Selected strategic and technical aspects}

\section{General feasibility}

Criteria for considering environmental PV surveillance as a supplementary approach in GPEI were published in WHO Guidelines for Environmental Poliovirus Surveillance [9]. The Guidelines describe situations when it might be necessary to consider ENV as a supplementary approach, contain principles for selecting sampling sites, propose methods for sample processing, and suggest possible programme responses to PV detection in sewage. Further, the Guidelines state the need for identification of resources for sample collection and transport to a dedicated laboratory as well as availability of trained staff, necessary equipment and supplies in the laboratory. Two main factors limit the wider application of this approach; first, the lack of sewer networks in most of the world poses a challenge for identifying representative sampling sites, and second, the analysis of the samples with current techniques in a laboratory is resource-demanding. One of us (E.dG.) has calculated for GPEI that a 'start-up' cost of equipment would be about US\$ 50000 for a laboratory already involved in PV isolation from clinical specimens, and the cost of supplies for analysing 100 specimens about US\$33000. Sample collection is not expensive but training and staff salaries have to be taken in account in the planning. According to an almost 10-year experience in Egypt, processing and analysis of 100 sewage samples in the laboratory requires trained staff at about the same level as for processing and analysis of stools from 200 AFP cases with two specimens from each case (L. El-Bassioni, personal communication).

\section{Selecting sampling sites}

For systematic environmental PV surveillance it is optimal but not obligatory that most, if not all, households are equipped with water closets connected to a converging sewer network (Fig. 1) allowing collection of downstream samples that represent a large number of people living in the catchment area. The number of people living in the catchment area affects the sensitivity of PV detection in a population in two ways: by increasing the area it is possible to monitor more people with fewer samples. On the other hand, this increase is likely to diminish the sample sensitivity, i.e. capacity to detect small numbers of PV excretors in the population as the increasing number of non-excretors may dilute the virus to below the limits of detection. In practice, the size of the source population in established systems where WPV and VDPV have been detected has varied from tens of thousands to a few millions.

\section{Modelling of factors affecting sensitivity}

Ranta and co-workers [11] have presented a mathematical model visualizing how different factors influence the proportion of excreted PV which can be recovered at a downstream sampling site, and thus sample sensitivity or population sensitivity, respectively, in ENV (Fig. 2). The model also demonstrated how different factors affecting the sensitivity of the approach are inter-related, e.g. how less than optimal virus detection sensitivity could be compensated for by collecting larger volumes for analysis, and revealed, unsurprisingly, that for detection of emerging 


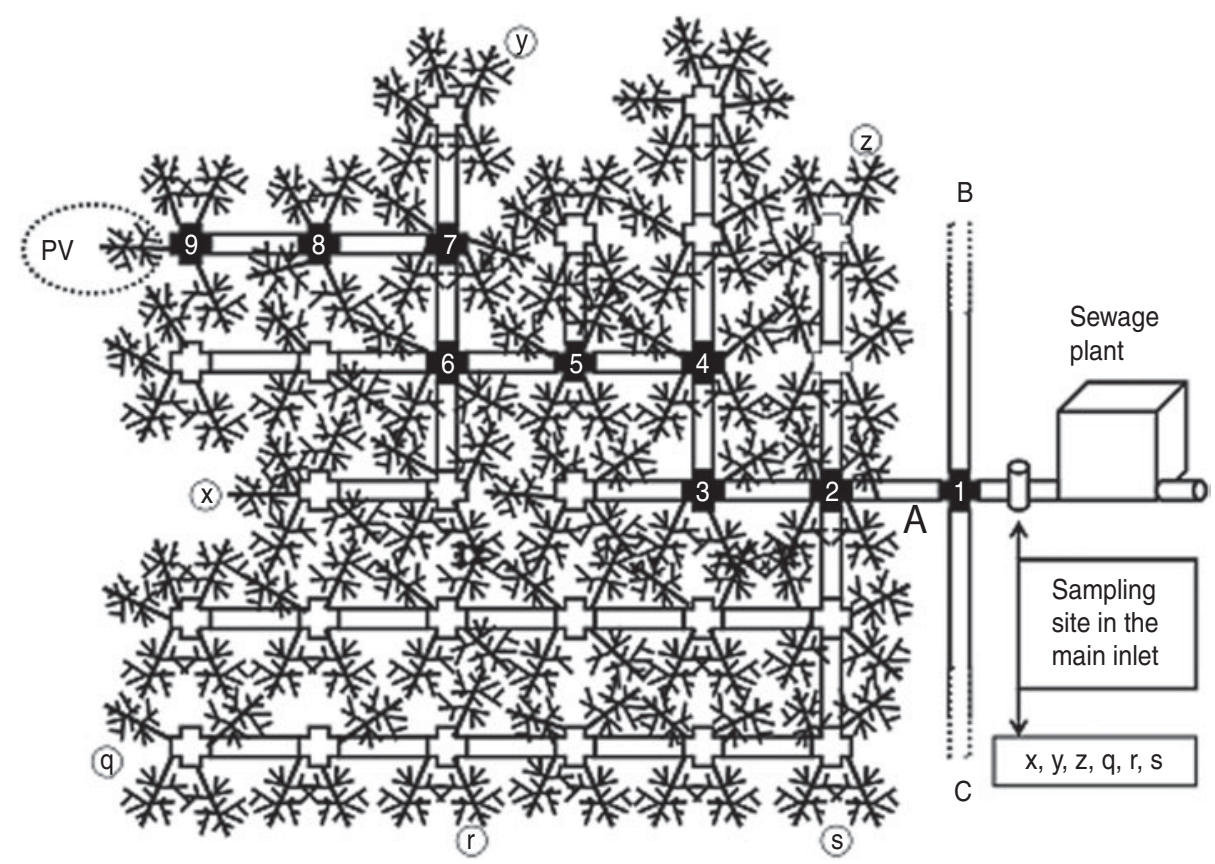

Fig. 1. Use of converging sewage systems for monitoring poliovirus circulation. A schematic picture of a sewer network with small branches starting from a single house or group of houses, joining stepwise to larger sewer branches, and finally ending in a sewage treatment plant. The inlet to the plant is the primary sampling site for monitoring the population served by this network. Two specific features are visualized. (1) Small circles with lower-case letters represent different viruses circulating in different locations of excretors throughout the catchment area of main sewer line A. At the main sampling site these viruses, plus those putatively derived from main sewers B and C, may appear as a complex mixture. (2) The dashed circle labelled PV represents a situation where a single or small group of poliovirus (PV) excretor(s) live in a geographically restricted subregion of the catchment area. Decreasing numbers at successive sites of network junctions (§) along the trunk lines leading towards the processing plant indicate a decreasing likelihood of PV detection as the concentration of virus is diluted by wastewater from converging lines. In case wild type or vaccine-derived poliovirus is detected at the primary sampling site, the same numbers reflect the order and positioning for secondary (and stepwise forward) sampling sites that are necessary in order to reveal the PV excretor(s).

outbreaks of virus circulation, frequently repeated sampling is critical. Applied at the standard bi-weekly analysis of 1 litre of sewage for PV in the Greater Helsinki Region representing about 700000 inhabitants, the model predicted that an emerging PV circulation would have been detected within a few months or at least as quickly as by using optimal AFP surveillance. This prediction was in reasonable agreement with conclusions drawn from experiments testing the fate of a known amount of attenuated PV flushed into the sewerage system. The authors of the report of that trial [12] calculated that by analysing a single 400-ml specimen, PV circulation could have been detected if about 100 individuals were infected with PV.

\section{Optimization of sample collection schedules}

When monitoring large populations served by a complex sewer network, simple daily grab samples are likely to be sufficient as transport of any input virus bolus is partially delayed, and virus concentration at the sampling site is not affected by toilet-use frequency of the source population [12]. The closer the sampling site is to the source, the higher the probability of detection. However, if the sampling site is very close to a suspect population, samples may not be sufficiently dispersed and a composite sampling system, i.e. daily pooled samples composed of hourly collected aliquots, may be necessary in order not to miss the peak virus concentrations in the sewage.

\section{Detection of rare viruses from a complex mixture}

Various techniques have been used for sewage sample collection and processing. In most cases, the processing includes a concentration step, up to 100 -fold. It is obvious that the exploited approaches differ in sensitivity but no systematic comparative studies have been published, so the relative specificities and efficacies of different methods will not be commented upon here. Thus far, attempts to directly use molecular detection methods for analysing sewage 


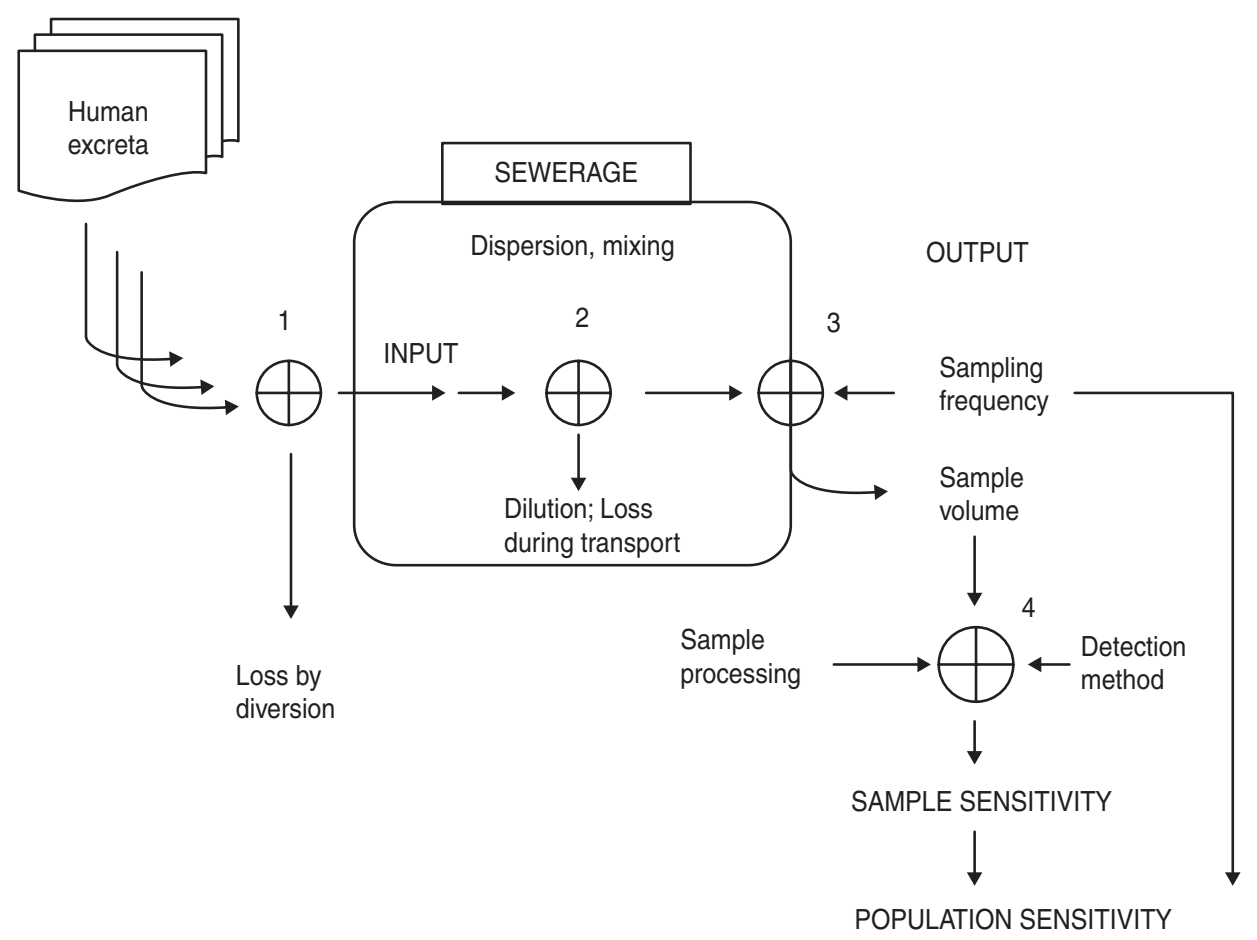

Fig. 2. Factors influencing recovery of poliovirus (PV) from the sewage system. Circles with a cross represent steps where uncontrollable factors (steps 1 and 2) or controllable factors (steps 3 and 4) may affect the end results. PV aggregates are dispersed and fluctuations of concentrations smoothed by the complexity of the sewage systems that include turbulence from high flow rates and converging streams from multiple trunk lines at branch points and in some cases from pumping stations that lift sewage upwards from low-lying areas to allow continuation of gravitational flow to the processing plant. All PVs excreted by the source population will not be readily detectable at the sampling site as some will be inactivated during transit, faecal matter from disposable baby diapers is disposed elsewhere among solid waste, and the complexity of the sewerage may result in retention of some of the input virus preventing it from ever reaching the sampling site or delaying its passage so that it arrives after the sample is taken.

concentrates have not been very successful presumably because, among other things, one or more components of the sample may inhibit the reverse transcriptase or DNA polymerase used in the methods. It is hoped that these obstacles can be eliminated in the near future because using cell culture for PV detection is slow, labour-intensive, and expensive.

In the cell culture approach, a PV-selective cell line (L20B) is used to avoid masking by other cytopathic viruses especially human non-polio enteroviruses present in the sample. Regular immunizations or campaigns with oral poliovirus vaccine (OPV) in the target population may result in abundance of Sabinlike (SL) PVs in the sewage, which have the potential to interfere with the detection of lower concentrations of WPV or VDPV potentially present in the same samples. Use of multiple parallel cell vials with standard monolayer culture has coped with this problem surprisingly well, as described below for Egypt and India (Table 3). In Egypt, increasing the number of parallel vials inoculated with a given sample usually increased the number of different virus strains isolated, i.e. improved the sample sensitivity. At the same time, it limited the number of samples that could be analysed with the same laboratory resources, i.e. possibly decreased the population sensitivity of detecting virus circulation [13]. While the laboratory workload per sewage sample is several times that caused by a faecal sample, it should be borne in mind that with sewage samples it is possible to monitor a large group of people rather than an individual.

In Israel, another cell culture-based approach has been used. When OPV was used alone, or in combination with inactivated poliovirus vaccine (IPV), the sewage surveillance protocol employed plaque isolation of all PVs in a sewage sample on L20B cells followed by selection for non-Sabin PV from among all PVs plaques in each sewage sample by a 5-day temperature selection at $40^{\circ} \mathrm{C}$ of each plaque on HEp2C cells, and subsequent molecular analysis of the heatresistant strains $[14,15]$. This 5-day temperature selection step was discontinued when the detection of 
Table 3. Examples of variation of isolation results from five parallel L20B cell vials (modified from a table originally published in [13])

\begin{tabular}{|c|c|c|c|c|c|}
\hline \multirow{2}{*}{$\begin{array}{l}\text { Sample } \\
\text { ID no. }\end{array}$} & \multicolumn{5}{|c|}{ Isolation results for individual vials } \\
\hline & Vial no. 1 & Vial no. 2 & Vial no. 3 & Vial no. 4 & Vial no. 5 \\
\hline 56 & WPV1 & SL2 & - & SL2 & SL2 \\
\hline 82 & - & $\mathrm{WPV} 1+\mathrm{SL} 2+\mathrm{SL} 3$ & $\mathrm{SL} 2+\mathrm{SL} 3$ & - & SL2 \\
\hline 93 & - & WPV1 + SL2 & SL2 & - & - \\
\hline 99 & SL3 & WPV1 + SL3 & $\mathrm{DR} 1+\mathrm{SL} 2+\mathrm{SL} 3$ & - & WPV11+SL3 \\
\hline 100 & SL1 & - & SL3 & $\mathrm{SL} 1+\mathrm{SL} 2$ & SL2 \\
\hline 107 & SL3 & SL2 & SL1 & SL3 & NSL1 \\
\hline 109 & SL1 & WPV1 & WPV1 + SL1 & - & WPV1 \\
\hline 111 & - & - & - & SL3 & WPV1 \\
\hline 112 & SL2 + SL3 & $\mathrm{WPV} 1+\mathrm{SL} 2+\mathrm{SL} 3$ & $\mathrm{WPVL} 1+\mathrm{SL} 2+\mathrm{SL} 3$ & SL2 & SL2 + SL3 \\
\hline
\end{tabular}

-, No cytopathic effect; WPV, wild type poliovirus=non-Sabin-like; SL, Sabin (OPV)-like poliovirus; DR, double reactive poliovirus (inconclusive result in the intratypic-differentiation test).

Numbers after WPV, SL, and DR indicate the serotype of poliovirus.

vaccine viruses in the samples stopped after Israel switched to exclusive use of IPV in 2006.

\section{Quality assurance of a non-standardized approach}

While the virus detection from ENV specimens by cell culture isolation and characterization of possible PV isolates follows the standard principles and is carried out by the laboratory network designed for AFP surveillance, the sample processing methods have not been standardized. A suggested quality assurance standard for sewage surveillance is that at least $30 \%$ of all sewage samples at any sampling site should be positive for non-polio enteroviruses [9]). The figure may depend on various factors but experience from routine use indicates that it is easy to reach in different countries. In both Finland and Israel the percentage has been about $80 \%$ in recent years (routine surveillance data of THL and Central Virology Laboratory, respectively).

In the following sections we will briefly discuss experiences from the use of ENV in attempts to answer selected specific epidemiological questions and review some relevant observations from routine use of ENV in PV surveillance.

\section{Use of ENV as a tool to solve specific epidemiological questions}

Studies aimed at assessing the extent of a polio outbreak in a population

PV infections occur at least 100 -fold more frequently than paralytic cases caused by the infection. In a routine AFP reporting system as well as under passive notification of paralytic cases of poliomyelitis, information about the circulation of the causative PV may underestimate the geographical range, the subpopulations affected and the length of circulation. As ENV is not case-driven but is capable of revealing any type of infections, it has been used to complement the picture of outbreaks based on reported clinical cases. Three major series of observations in this category will be briefly described, corresponding to outbreaks in Finland (1984), Israel (1988) and The Netherlands (1992).

Finland had been free of WPV for 20 years when an outbreak of WPV3 took the country by surprise in late $1984[16,17]$. As several contacts of the index case also excreted a similar WPV3, ENV was utilized to assess the extent of virus circulation, first in the Greater Helsinki region and later throughout the country [18]. The epidemic WPV3 strain was found in 18/26 samples collected in December 1984-January 1985 in 13/15 different locations in the Helsinki region and in 14/21 sites elsewhere in the country. Most of the latter locations were sampled only once and most of the respective districts had shown no PV infectionassociated cases of AFP at all [18]. In other words, under these conditions ENV provided crucial supplementary information indicating a wide geographical extent of WPV 3 circulation in the country.

In Israel, the anti-polio immunization programme towards the end of 1980s was mainly based on the use of OPV but included a small region of the country with exclusive enhanced-potency IPV administration. An outbreak discovered in 1987 was caused by WPV1 with cases in different parts of the country without a 
simple correlation to the previous immunization history of the respective population [19]. Here also, the response after the detection of the outbreak included establishment of monthly ENV extending into wider regions of the country. WPV1 was detected in 6/197 sewage samples collected during the outbreak in 1987-1988. As in Finland, ENV detected the epidemic virus in areas of Israel, where no paralysed cases were reported [19, 20].

In The Netherlands, in 1992-1993 an outbreak of poliomyelitis was detected in the unvaccinated minority group of the Dutch population that refuses vaccination for religious reasons [21]. Neither cases nor virus were detected in the majority of the population immunized with IPV only. Overall, WPV3 was detected in 23/269 sewage samples collected at 120 locations spread over the whole country. The epidemic virus was found only in sewage derived from villages mainly inhabited by the unvaccinated people, but not in regions where the rest of the regularly immunized Dutch population lived. In some locations, the virus was detected in sewage a few weeks before the first cases of poliomyelitis were reported in the same region, demonstrating the potential power of ENV to herald epidemics and in outbreak investigations [22].

\section{Search for putative WPV reservoirs and monitoring the decline of WPV transmission}

Molecular epidemiological analysis of PV strains isolated from successive cases in a region occasionally shows 'jumps' of evolution in a given lineage. This is interpreted as indicating either suboptimal AFP surveillance resulting in missed cases, or, importation of the virus from an unknown reservoir of virus transmission. Targeted ENV could be used as a supplementary tool to attempt to discover which of the two alternatives is likely to be correct.

At the end of 1990s, Egypt was faced with suspected gaps in PV surveillance. ENV was initiated in 2000 with sewage samples collected from inlets of sewage plants in two provinces and later extended to cover most provinces of the country. Rather than uncovering a presumed distinct reservoir region, ENV rapidly showed that WPV1 was circulating in many regions of the country, not only in those provinces which had shown paralytic cases in recent years, but in several others as well including some distant ones [23]. Interestingly, in 2002 ENV in the Gaza district detected importation of PV related the Egypt ENV lineages [15]. These observations resulted in intensified immunization campaigns and improved AFP
Table 4. Wild poliovirus (WPV) transmission in Egypt monitored with acute flaccid paralysis (AFP) surveillance vs. environmental surveillance in 2001-2009

\begin{tabular}{|c|c|c|c|c|}
\hline \multirow[b]{2}{*}{ Year } & \multicolumn{2}{|c|}{ AFP cases } & \multicolumn{2}{|c|}{ Sewage samples } \\
\hline & $\begin{array}{l}\text { No. } \\
\text { tested }\end{array}$ & $\begin{array}{l}\text { No. with } \\
\text { WPV1 } \\
\text { (no. provinces) }\end{array}$ & $\begin{array}{l}\text { No. } \\
\text { tested }\end{array}$ & $\begin{array}{l}\text { No. with } \\
\text { WPV1 } \\
\text { (no. provinces) }\end{array}$ \\
\hline 2001 & 257 & $5(2)$ & 130 & $71(8)$ \\
\hline 2002 & 576 & $7(5)$ & 162 & $26(11)$ \\
\hline 2003 & 608 & $1(1)$ & 311 & $12(6)$ \\
\hline 2004 & 768 & $1(1)$ & 482 & $13(4)$ \\
\hline 2005 & 859 & 0 & 501 & $2(2)$ \\
\hline 2006 & 978 & 0 & 492 & 0 \\
\hline 2007 & 1070 & 0 & 458 & 0 \\
\hline 2008 & 1116 & 0 & 446 & $2 *$ \\
\hline $2009 \dagger$ & 1058 & 0 & 450 & 0 \\
\hline
\end{tabular}

* Two separate importations of WPV1 strains genetically unrelated to indigenous strains.

$\dagger$ Data up to 7 December.

surveillance throughout Egypt. The still continuing, intensive ENV in Egypt later revealed the last indigenous WPV1 case in January 2005, 1 year after the last paralytic case, and, in 2008, two introductions of WPV belonging to separate non-indigenous genotypes (Table 4). No associated paralytic cases were found in Egypt during follow-up investigations, but responsive precautionary SIAs were conducted [24].

In Colombia, ENV was used with success in the final stages of the eradication programme. Like the situation in Egypt, one of two genetic lineages of WPV isolated from samples collected in open canals in Cartagena was absent from poliomyelitis cases or from healthy contacts investigated using AFP surveillance [25].

In India, following the national launch of the GPEI, WPV transmission significantly declined and by the early 2000s was mainly restricted to the northern states of Uttar Pradesh and Bihar while, for instance, in the western coast island city of Mumbai, only sporadic cases occurred, mainly in the slum areas. In the absence of sewage networks, wastewater samples were collected from the open canals containing waste from populous areas [26] WPV strains were frequently isolated from samples even in the absence of concurrent paralytic cases in the corresponding population (Table 5). Phylogenetic analysis later showed that virus strains detected in these samples were rather diverse as regards their origin with representatives from the still endemic northern parts of India. Rather than 
Table 5. Wild poliovirus (WPV) and vaccine-derived poliovirus (VDPV) detection in sewage in Mumbai compared to results of acute flaccid paralysis (AFP) surveillance

\begin{tabular}{|c|c|c|c|c|c|c|c|c|c|}
\hline \multirow[b]{4}{*}{ Year } & \multicolumn{6}{|c|}{ Greater Mumbai region } & \multirow{2}{*}{\multicolumn{3}{|c|}{$\begin{array}{l}\text { All India } \\
\text { AFP surveillance }\end{array}$}} \\
\hline & \multicolumn{3}{|c|}{ Environmental } & \multicolumn{3}{|c|}{ AFP surveillance } & & & \\
\hline & \multirow{2}{*}{$\begin{array}{l}\text { No. of } \\
\text { sewage } \\
\text { samples }\end{array}$} & \multicolumn{2}{|c|}{ No. with WPV } & \multirow{2}{*}{$\begin{array}{l}\text { No. of } \\
\text { AFP } \\
\text { cases }\end{array}$} & \multicolumn{2}{|c|}{ No. with WPV } & \multirow{2}{*}{$\begin{array}{l}\text { No. of } \\
\text { AFP } \\
\text { cases }\end{array}$} & \multicolumn{2}{|c|}{ No. with WPV } \\
\hline & & Type 1 & Type 3 & & Type 1 & Type 3 & & Type 1 & Type 3 \\
\hline 2005 & 150 & 16 & 0 & 169 & 0 & 0 & 27049 & 62 & 4 \\
\hline 2006 & $45^{*}$ & 2 & 0 & 310 & 3 & 0 & 32194 & 648 & 28 \\
\hline 2007 & $105^{*}$ & 9 & 3 & 254 & 0 & 0 & 41516 & 88 & 6 \\
\hline 2008 & 159 & 2 & 30 & 296 & 0 & 1 & 45582 & 75 & 484 \\
\hline 2009 & 144 & $1 \dagger$ & $0 \dagger$ & 363 & 0 & 0 & 50404 & 80 & 661 \\
\hline
\end{tabular}

* The laboratory processing environmental surveillance specimens was closed from mid-April 2006 to the end of April 2007 because of a fire.

$\dagger$ One VDPV strain of each serotype.

representing a local reservoir of PV circulation, repeated isolation of WPV in the slums thus appeared to be caused by repeated introductions of the strains along with people moving from the endemic regions to Mumbai. Along with the success of control of poliomyelitis in India, the number and the genetic diversity of virus strains isolated from the sewage in Mumbai has also decreased (routinely reported data provided to the GPEI). WPV detection in the Mumbai sewage is taken into account by local authorities in planning SIAs. WPV has also been detected in sewage in Lucknow, a city situated in a high-risk area in northern India, during a research project between 2004 and 2006, and demonstrated silent circulation of WPV in the absence of detected poliomyelitis cases in that city [27]. Since 2010, ENV is also being used as a supplementary approach in Delhi.

\section{Evaluation of emerging transmission of $O P V$-derived viruses after immunization campaigns or a switch to IPV alone}

It is generally thought that possible transmission chains after OPV administration are very short and this is indeed likely to be true in OPV immunized populations with good herd immunity. Recent circulating VDPV (cVDPV) outbreaks have, however, raised concern that, under certain circumstances, especially following decreased OPV coverage [28], the OPV strains have the potential to cause sustained transmission and paralytic disease [29-39]. A widely held view is that IPV immunization does not effectively protect against PV replication in gut mucosa, and hence, a switch from OPV to IPV alone might result in a situation where OPV-derived viruses from the last period of use, from chronically infected persons, or imported from other locations, might be able to be transmitted. Several observational studies provide evidence against this suspicion and, in some situations, ENV has been utilized to gather this evidence (Table 6). Many industrialized countries have successfully switched from routine OPV immunization, or from combined OPV-IPV schedules to IPVonly programmes. Such countries generally rely on routine AFP surveillance or on passive case notification only. No signs of emerging transmission of OPV-related viruses have been reported.

Thus, so far all available data support the view that it is safe to switch from OPV to IPV in industrialized countries with high polio immunization coverage. However, there is a question about whether the same will be true in developing countries with high population density and poor sanitation. To evaluate this question, a study was designed and has been implemented on the island of Java, Indonesia, to monitor the range of genetic drift of PV strains detected in the environment before and after a switch from OPV to IPV in Yogjakarta province. The switch took place in 2007, and to date there has been no evidence of circulating OPV-derived strains (R. Sutter et al., unpublished data).

Detection of importations of $O P V$ strains, WPV and VDPV by routine ENV

Both Finland and Israel have used ENV as the main approach to PV surveillance for decades. In addition, several countries are currently using regular ENV as a 
Table 6. Environmental surveillance in documentation of short-lived oral poliovirus vaccine (OPV) circulation after cessation of $O P V$ administration

\begin{tabular}{lllll}
\hline \hline $\begin{array}{l}\text { Country, year of } \\
\text { OPV cessation }\end{array}$ & Mode of OPV use & $\begin{array}{l}\text { SL strains } \\
\text { frequently in } \\
\text { sewage, months } \\
\text { after switch }\end{array}$ & $\begin{array}{l}\text { Last SL isolate, } \\
\text { months after } \\
\text { switch }\end{array}$ & Ref. \\
\hline Finland, 1985 & $\begin{array}{c}\text { 5-week campaign; coverage } \\
\begin{array}{c}95 \% \text { of all age groups } \\
\text { Annual National Immunization } \\
\text { Day campaigns }\end{array}\end{array}$ & 3 & 6 & {$[18]$} \\
Cuba, 1997-1998 & 2 & 4 & {$[40]$} \\
New Zealand, 2002 & Regular childhood immunization & 3 & 12 & {$[41]$} \\
\hline \hline
\end{tabular}

SL, Sabin-like poliovirus strain.

supplementary approach in their national PV surveillance.

In countries using OPV in regular immunizations, PV SL strains are frequently isolated from sewage. IPV is exclusively used for polio immunizations in Finland, but annually millions of tourists travel to or from neighbouring Estonia and/or Russia where OPV has been used until very recently. About 60 samples are collected annually in Finland and the sampling sites cover about $20 \%$ of the population. While not a single PV was isolated from the Finnish sewage samples between autumn 1985 and 2005, at least one SL $\mathrm{PV}$ has been isolated from the samples annually since 2006 (routine data of THL). Similarly, ENV has detected OPV strains in sewage samples in another exclusive IPV country, The Netherlands (routine data of RIVM), suggesting that ENV might also readily detect WPV importations. Indeed, WPV importations have been detected in the absence of clinical cases in Israel and the Gaza district in spite of vaccine coverage above $95 \%$ and use of OPV in regular immunizations [14, 15].

The catchment areas in Israel currently serve $30-40 \%$ of the population and since $1989,>25$ sites were sampled monthly between 1989 and 2001, and $>11$ sites from 2002 to the present. Silent infections, i.e. in the absence of AFP cases, of WPV1, primarily in the Gaza district, were documented by ENV between May and September 1991, between October 1994 and June 1995, and in December 1996 [14]. Phylogenetic analyses of the WPV1 isolated from sewage in the Gaza district in 1994-1995 were consistent with local circulation of one of three WPV1 lineages introduced into the Gaza district from Egypt [14]. Routine monthly ENV during 1994-1995 also documented the interruption of this silent transmission by a subregional vaccination campaign conducted in response to the finding $[14,15]$.

Highly diverged neurovirulent VDPVs have been isolated by ENV from many geographical locations such as Egypt, Greece, Haiti, India, South Africa and Switzerland [33, 42-46]. Two independent epidemiological sources of type 2 VDPV have been identified in central Israel. Based on the rate of accumulation of single nucleotide substitutions, one of the sources was exposed to OPV around 1988, and 47 isolates from 32 sewage sample isolates from this source have been recovered from sewage between 1998 and 2010 [47, 48] (L. M. Shulman, unpublished data). It is noteworthy that the ENV protocol used in Israel was capable of detecting ambiguous VDPVs (aVDPVs) at a time when sewage also contained OPV from national vaccination [47]. Similarly, mutually related, highly diverged type 3 aVDPVs have been isolated from sewage in Estonia between 2002 and 2008, as well as two similar type 2 aVDPVs in 2008 and 2009, in the presence of OPV strains [49] (M. Roivainen et al., unpublished data).

Several aVDPVs were found in sewage in Slovakia in 2003-2005 [50]. This country has been using supplementary ENV with samples collected at 47 sites dispersed throughout the country, usually at 2-month intervals. Starting from the capital Bratislava in April 2003 , more than 100 related type 2 aVDPV strains with vast sequence divergence from Sabin 2 strain have been characterized (T. Hovi et al., unpublished data). All but two of the strains were found in sewage from a small town of Skalica, located $\sim 60 \mathrm{~km}$ north of the capital. With a retrospective 'walk' of sampling sites along the bifurcations of the sewage network (Fig. 2) the virus excretor(s) was located among $\sim 500$ people living in a few blocks of flats, but despite much 
effort the source of the strain could not be identified before the occurrence of the virus in the sewage samples completely stopped. Throughout the 2 years, the virus was neither found in clinical specimens from infectious disease patients or from immune-deficient individuals nor in sewage in locations other than Skalica, and initially in 2003 in Bratislava. It was therefore concluded, that virus excretion was highly local perhaps due to one single individual with possibly a few occasional contacts.

Since December 2008, several highly divergent VDPVs have been isolated intermittently from sewage specimens collected in Tampere, a city in the southern part of Finland. Interestingly, four out of five positive specimens contained VDPVs of more than one serotype and one specimen VDPV of all three serotypes. All VDPVs were highly divergent from the corresponding vaccine strains (similarities $<88 \%$ ) suggesting that they might be originally derived from a chronically infected, still unidentified, immunedeficient individual(s) [51].

On top of the threat of emerging cVDPV outbreaks under suboptimal OPV vaccination coverage [33-42], existence of immune-deficient long-term PV (iVDPV) excretors present a challenge to GPEI. Frequent isolation by ENV of vastly drifted aVDPV strains genetically resembling iVDPV [47, 49-51] suggests that long-term PV excretion is not limited to immunedeficient individuals known to the health system, or if known to the health system not recognized as chronically excreting PV.

\section{CONCLUDING REMARKS AND FUTURE VIEWS}

Experiences from several countries confirm that ENV can detect introduction and silent circulation of WPV and VDPV (Table 7), sometimes before any AFP cases occur. Likewise, ceasing PV transmission may be monitored with ENV with greater sensitivity than with AFP surveillance, as described above for Egypt and India. Further, ENV can be used to monitor the efficacy of immunization interventions when it is required. The list of countries routinely employing ENV currently includes Czech Republic, Egypt, Estonia, Finland, India, Israel, Japan, Latvia, The Netherlands, New Zealand, Pakistan, Russia, Slovakia, and Switzerland. In Finland and Israel, it is considered to be the main approach of PV surveillance. ENV also has a role in the new WHO strategy for intensified efforts to complete poliomyelitis eradication [3].
Proper programme responses to detection of WPV or VDPV by ENV depend on the actual observation and epidemiological situation but always include an alert to improve surveillance in all possible aspects [9].

With conventional laboratory techniques, the approach is rather labour and resource intensive. Simpler laboratory techniques and high-capacity equipment allowing for handling large workloads are perhaps needed in order to increase the use of this approach. Another significant factor limiting more wide-scale use of ENV is the fact that dwellings of most people living in developing countries are not connected to converging sewage networks. Under these conditions, although suitable environmental material is usually abundantly available, individual samples do not represent large numbers of people. Experience in India, however, has shown that careful selection of sampling sites representing open sewers in highly populated or high-risk areas can overcome this limitation. Significant resources are currently available to implement AFP surveillance in developing countries but such surveillance and investment may not be sustainable in the long term. In certain situations, ENV can provide an alternative approach for monitoring PV circulation, independent of the local healthcare infrastructure, with design of sample collection based on knowledge of the sanitary organization of the municipalities. Yet, it is clear that in order to provide useful information, ENV also requires dedicated resources, trained laboratory staff and close cooperation and coordination with municipal, regional and national authorities which should be guaranteed before the decision to start ENV is made.

After eradication of WPV transmission, the risk of re-emergence of polio remains extremely high as long OPV continues to be used routinely or in campaigns organized for any reason, and thus provides a potential source of circulating neurovirulent VDPV strains [35]. Environmental contamination with faecal effluents, increasing use of wastewater in agriculture, and declining PV population immunity are all likely future risk factors for ingestion and transmission of PVs [10]. ENV can be an important tool for shortening the response time between awareness of a PV reemergence event and response. ENV should be available and in place during the critical period between interruption of WPV transmission and certification of polio eradication, and, should continue into the posteradication and OPV cessation periods to monitor for the emergence of VDPVs, re-emergence of WPVs, or disappearance of all OPV-related strains. Research 
Table 7. Global detection of wild and vaccine-derived polioviruses in sewage waters in 1984-2010

\begin{tabular}{|c|c|c|c|c|c|c|c|}
\hline Category of virus & Country & City or region & Year & Serotype & Genotype* & Source of virus & Ref. \\
\hline \multirow[t]{15}{*}{ Wild poliovirus } & Colombia & Cartagena & 1991 & 1 & & Indigenous & [25] \\
\hline & Egypt & Multiple & 2000-2005 & 1 & NEAF & Indigenous & [23] \\
\hline & & Cairo & 2008 & 1 & WEAF-B & Imported, Sudan & [24] \\
\hline & & & & 1 & SOAS & Imported, India & [24] \\
\hline & Finland & Nationwide & 1984-1985 & 3 & & Imported & {$[16,18]$} \\
\hline & India & Lucknow & 2004-2006 & 1 & SOAS & Indigenous & {$[27]$} \\
\hline & & Mumbai & 2001-2009 & 1 & SOAS & Indigenous & [26] \\
\hline & & & & 3 & SOAS & Indigenous & [26] \\
\hline & Israel and Gaza & Multiple & 1987-1988 & 1 & NEAF & Imported & {$[14,20]$} \\
\hline & & Gaza & 1991-2002 & 1 & & Imported & [15] \\
\hline & The Netherlands & Multiple & 1992-1993 & 3 & & Imported & [22] \\
\hline & Pakistan & Karachi & 2009-2010 & 1 & & Indigenous & $\dagger$ \\
\hline & & & 2009-2010 & 3 & & Indigenous & $\dagger$ \\
\hline & & Lahore & 2009 & 1 & & Indigenous & $\dagger$ \\
\hline & Switzerland & Geneva & 2007 & 1 & WEAF-B & Imported, Sudan & [46] \\
\hline \multirow{15}{*}{$\begin{array}{l}\text { Vaccine-derived } \\
\text { poliovirus }\end{array}$} & Egypt & & & & & & [24] \\
\hline & Estonia & Tallinn & 2002,2008 & 3 & & & [49], $\ddagger$ \\
\hline & & & 2008, 2009 & 2 & & & $\$$ \\
\hline & Finland & Tampere & 2008 & 1,2 & & & {$[51], \ddagger$} \\
\hline & & & 2009-2010 & $1,2,3$ & & & {$[51], \ddagger$} \\
\hline & Greece & Athens & 1997 & 1 & & & [43] \\
\hline & Hispaniola & & 2000 & 1 & & & [44] \\
\hline & India & Mumbai & 2009 & 1 & & & $\S$ \\
\hline & & & & 3 & & & $\S$ \\
\hline & Israel & & 1998-2010 & 2,1 & & & [47], $\|$ \\
\hline & Slovakia & Bratislava & 2003 & 2 & & & {$[50], \ddagger$} \\
\hline & & Skalica & 2003-2005 & & & & \\
\hline & South Africa & & 2001-2003 & 1,2 & & & [45] \\
\hline & Switzerland & Zurich & 2008 & 1 & & & $\dagger$ \\
\hline & & Geneva & 2008 & 2 & & & $\dagger$ \\
\hline
\end{tabular}

* Relevant to the wild viruses only. Four first digits in the genotype name refer to the geographical origin of the genotype; NE, north-eastern; WE, western; SO, southern; AF, Africa; AS, Asia.

$\dagger$ Routine notification to WHO.

$\$$ National Institute for Health and Welfare (THL), Finland (unpublished observations).

$\S$ Environmental Research Centre (ERC), India (unpublished observations).

\| Central Virological Laboratory (CVL), Ministry of Health, Israel (unpublished observations).

and development to improve and simplify the technology required to demonstrate $\mathrm{PV}$ in environmental specimens should be encouraged and allocated with sufficient resources in order to make this approach more widely available. An added benefit is that the methodology and infrastructure of ENV can also be adapted to monitor for non-polio enterovirus and other environmental pathogens.

\section{ACKNOWLEDGEMENTS}

We are grateful to members of the environmental virology teams at our home laboratories, including Soile Blomqvist and Mirja Stenvik at the National
Institute for Health and Welfare (THL), Finland; Yossi Manor, Ella Mendelson and Danit Sofer at the Central Virological Laboratory (CVL), Israel; Ron Altena and Edin Jusic at the National Institute of Public Health and the Environment (RIVM), The Netherlands; and Sushmitha Shetty at the Enterovirus Research Centre (ERC), India. Roland Sutter and Olen Kew are acknowledged for providing unpublished data for our use.

\section{DECLARATION OF INTEREST}

One of the authors (E.M.dG.) is employed by the WHO and all other authors are working in member 
laboratories of the WHO Polio Laboratory Network, which have received partial support from the WHO.

\section{REFERENCES}

1. World Health Assembly. Global eradication of poliomyelitis by the year 2000. Geneva, Switzerland: WHA resolution no. WHA41.28, 1988.

2. WHO. Field guide for supplementary activities aimed at achieving polio eradication, publication no. WHO/ EPI/GEN/95.1. Geneva: World Health Organization, 1995.

3. The Global Polio Eradication Strategic Plan, 2010-2012. (http://www.polioeradication.org/content/ publications/GPEI.StrategicPlan.2010-2012.ENG.May. 2010.pdf). Accessed 6 October 2010.

4. Rico-Hesse R, et al. Geographic distribution of wild poliovirus type 1 genotypes. Virology 1987; 160: 311-322.

5. Anon. Progress towards interrupting wild poliovirus transmission worldwide, 2008. Weekly Epidemiological Records 2009: 84; 110-116.

6. Roberts L. Polio eradication. Looking for a little luck. Science 2009; 323: 702-705.

7. Anon. Resurgence of wild poliovirus types 1 and 3 in 15 African countries, January 2008-March 2009. Weekly Epidemiological Records 2009; 84: 133-140.

8. Anon. CDC Update on vaccine-derived polioviruses worldwide, January 2008-June 2009. Mobridity and Mortality Weekly Report 2009; 58: 1002-1006.

9. WHO. Guidelines for environmental surveillance of poliovirus circulation. World Health Organization, Department of Vaccines and Biologicals, 2003. (http:// www.who.int/vaccines-documents/DoxGen/H5-Surv. htm). Accessed 6 October 2010.

10. Dowdle W, et al. Containment of polioviruses after eradication and OPV cessation: characterizing risks to improve management. Risk Analysis 2006; 26: 1449-1469.

11. Ranta J, Hovi T, Arjas E. Poliovirus surveillance by examining sewage water specimens: studies on detection probability using simulation models. Risk Analysis 2001; 21 : 1087-1096.

12. Hovi $\mathbf{T}$, et al. Poliovirus surveillance by examining sewage specimens. Quantitative recovery of virus after introduction into sewerage at remote upstream location. Epidemiology and Infection 2001 ; 127 : 101-106.

13. Hovi T, et al. Environmental surveillance of wild poliovirus circulation in Egypt - balancing between detection sensitivity and workload. Journal of Virological Methods 2005; 126: 127-134.

14. Manor Y, et al. Detection of poliovirus circulation by environmental surveillance in the absence of clinical cases in Israel and the Palestinian authority. Journal of Clinical Microbiology 1999; 37: 1670-1675.

15. Manor Y, et al. Advanced environmental surveillance and molecular analyses indicate separate importations rather than endemic circulation of wild type 1 poliovirus in Gaza district in 2002. Applied and Environmental Microbiology 2007; 73: 5954-5958.

16. Hovi T, et al. Outbreak of paralytic poliomyelitis in Finland: widespread circulation of antigenically altered poliovirus type 3 in a vaccinated population. Lancet 1986; 1: 1427-1432.

17. Kinnunen E, Hovi T, Stenvik M. Outbreak of poliomyelitis in Finland in 1984. Description of nine cases with persisting paralysis. Scandinavian Journal of Infectious Diseases 1986; 18: 15-18.

18. Pöyry T, Stenvik M, Hovi T. Viruses in sewage waters during and after a poliomyelitis outbreak and subsequent nationwide oral poliovirus vaccination campaign in Finland. Applied and Enviromental Microbiology 1988; 54: 371-374.

19. Slater PE, et al. Poliomyelitis outbreak in Israel in 1988: a report with two commentaries. Lancet 1990; 335: 1192-1195; discussion 1196-1198.

20. Shulman LM, et al. Resolution of the pathways of poliovirus type 1 transmission during an outbreak. Journal of Clinical Microbiology 2000; 38: 945-952.

21. Oostvogel PM, et al. Poliomyelitis outbreak in an unvaccinated community in The Netherlands, 1992-93. Lancet 1994 ; 344: 665-670.

22. van der Avoort HG, et al. Isolation of epidemic poliovirus from sewage during the 1992-3 type 3 outbreak in The Netherlands. Epidemiology and Infection 1995; 114: 481-491.

23. El Bassioni L, et al. Prolonged detection of indigenous wild polioviruses in sewage from communities in Egypt. American Journal of Epidemiology 2003; 158: 807-815.

24. World Health Organization. Global detection of wid and vaccine-derived polioviruses, January 2008-June 2009. Weekly Epidemiological Records 2009; 36: 366-371.

25. Tambini G, et al. Direct detection of wild poliovirus circulation by stool surveys of healthy children and analysis of community wastewater. Journal of Infectious Diseases 1993; 168: 1510-1514.

26. Deshpande JM, Shetty SJ, Siddiqui ZA. Environmental surveillance system to track wild poliovirus transmission. Applied and Environmental Microbiology 2003; 69: 2919-2927.

27. Chowdhary R, Dhole TN. Interrupting wild poliovirus transmission using oral poliovirus vaccine: environmental surveillance in high-risks area of India. Journal of Medical Virology 2008; 80: 1477-1488.

28. Korotkova EA, et al. Retrospective analysis of a local cessation of vaccination against poliomyelitis: a possible scenario for the future. Journal of Virology 2003; 77: 12460-12465.

29. Kew O, et al. Outbreak of poliomyelitis in Hispaniola associated with circulating type 1 vaccine-derived poliovirus. Science 2002; 296: 356-359.

30. Anon. Paralytic poliomyelitis in Madagascar, 2002. Weekly Epidemiological Records 2002; 77: 241-242.

31. Dowdle WR, et al. Polio eradication: the OPV paradox. Reviews in Medical Virology 2003; 13: 277-291.

32. Shimizu H, et al. Circulation of type 1 vaccine-derived poliovirus in the Philippines in 2001. Journal of Virolology 2004; 78: 13512-13521. 
33. Kew OM, et al. Circulating vaccine-derived polioviruses: current state of knowledge. Bulletin of World Health Organization 2004; 82: 16-23.

34. Liang X, et al. An outbreak of poliomyelitis caused by type 1 vaccine-derived poliovirus in China. Journal of Infectious Diseases 2006; 194: 545-551.

35. Tebbens RJ, et al. Risks of paralytic disease due to wild or vaccine-derived poliovirus after eradication. Risk Analysis 2006; 26: 1471-1505.

36. Rakoto-Andrianarivelo $\mathbf{M}$, et al. Reemergence of recombinant vaccine-derived poliovirus outbreak in Madagascar. Journal of Infectious Diseases 2008; 197: 1427-1435.

37. Estívariz CF, et al. A large vaccine-derived poliovirus outbreak on Madura Island - Indonesia, 2005. Journal of Infectious Diseases 2008; 197: 347-354.

38. Jenkins HE, et al. Implications of a circulating vaccinederived poliovirus in Nigeria. New England Journal of Medicine 2010; 362: 2360-2369.

39. Gumede N, et al. Identification of vaccine-derived polioviruses (VDPVS) in the DRC from 2005 to 2010. Communicable Diseases Surveillance Bulletin 2010; 8: 43-45.

40. Más Lago $\mathbf{P}$, et al. Poliovirus detection in wastewater and stools following an immunization campaign in Havana, Cuba. International Journal of Epidemiology 2003; 32: 772-777.

41. Huang QS, et al. Persistence of oral polio vaccine virus after its removal from the immunization schedule in New Zealand. Lancet 2005; 366: 394-396.

42. Kew OM, et al. Vaccine-derived polioviruses and the endgame strategy for global polio eradication. Annual Reviews of Microbiology 2005; 59: 587-635.
43. Dedepsidis E, et al. Retrospective characterization of a vaccine-derived poliovirus type 1 isolate from sewage in Greece. Applied and Environmental Microbiology 2007; 73: 6697-6704.

44. Vinjé $\mathbf{J}$, et al. Isolation and characterization of circulating type 1 vaccine-derived poliovirus from sewage and stream waters in Hispaniola. Journal of Infectious Diseases 2004; 189: 1168-1175.

45. Pavlov DN, et al. Prevalence of vaccine-derived polioviruses in sewage and river water in South Africa. Water Research 2005; 39: 3309-3319.

46. Bundesamt fur Gesundheit. Communicable diseases. European Region of World Health Organization free of wild virus-induced poliomyelitis for five years [in German]. Bulletin BAG 2007; 46: 824-826.

47. Shulman LM, et al. Neurovirulent vaccine-derived polioviruses in sewage from highly immune populations. PLoS ONE 2006; 1: e69.

48. Shulman LM, et al. Type 2 polio still in our midst. Science 2009; 324: 334.

49. Blomqvist S, et al. Characterization of a highly evolved vaccine-derived poliovirus type 3 isolated from sewage in Estonia. Journal of Virology 2004; 78: 4876-4883.

50. Cernáková B, et al. Isolation of vaccine-derived polioviruses in the Slovak Republic. European Journal of Clinical Microbiology \& Infectious Diseases 2005; 24: 438-439.

51. Roivainen M, et al. Highly divergent neurovirulent vaccine-derived polioviruses of all three serotypes are recurrently detected in Finnish sewage. Eurosurveillance $2010 ; \mathbf{1 5}$. 